\title{
HR-MRI of the Breast at Intervals of Maximum 24 Months: Influence on Tumor Stage at the Time of Diagnosis
}

\author{
Uwe Fischer $^{1 *}$, M Hollstein ${ }^{1}$, S Luftner-Nagel ${ }^{1}$, F Baum $^{1}$ and S Wienbeck ${ }^{2}$ \\ ${ }^{1}$ Diagnostic Breast Care Center Goettingen, Germany \\ ${ }^{2}$ Department of Interventional and Diagnostic Radiology, University of Göttingen, Germany
}

*Corresponding author: Uwe Fischer, Diagnostic Breast Care Center Goettingen, Bahnhofsallee 1d, 37081, Goettingen, Germany

\begin{abstract}
Purpose: To define the value of repetitive performed exclusive MRI of the breast at intervals of maximum 24 months in asymptomatic women and patients regarding the tumor size and stage at the time of diagnosis.

Material and methods: Women with normal MRI of the breast upon initial examination and subsequent breast MRI within intervals of maximum 2 years were included. Benign and malignant lesions as well as the tumor stages were reported and analysed.

Results: $8.975 \mathrm{MRI}$ were performed in 2333 women. 6642 exams were subsequent MRI (2.85 follow-up MRI/woman). Within the examination intervals, 290 (4.37\% per follow up exam) lesions categorized as MR-BIRADS 4/5 were found in 272 patients. All of these findings were clarified by percutaneous biopsy. Histology revealed benign findings (B1/B2) in 151/290 (52.1\%), B3-lesions in 24 (8.3\%), and malignant tumors (B5a/B5b) in 115 cases $(39.6 \%)$. Open biopsy revealed an intraductal carcinoma in $36 / 115$ cases (G1: 11, G2: 16, G3: 9), and an invasive breast cancer in $79 / 115$ cases. Tumor stages of the invasive carcinoma were pT1a (17/79; 21.5\%), pT1b (39/79, 49.4\%), and pT1c $(22 / 79 ; 27.8 \%)$. Only one invasive cancer was classified as stage pT2. The average size of all invasive tumors was 9.0 $\mathrm{mm}$. Rate of nodal negative invasive cancer was $95.9 \%$. Rate of interval carcinomas was less than $2 \%$.

Conclusion: The performance of repetitive MRI of the breast at intervals of maximum 24 months allows the reliable detection of breast cancer at an early stage (pTis, pT1a, pT1b, pN0) with an excellent prognosis.
\end{abstract}

\section{Keywords}

MRI of the breast, Mammography, Ultrasound, Breast cancer

\section{Introduction}

Tumor size, the histology and immunhistochemical parameters as well as the lymph node status at the time of diagnosis have a relevant influence on the prognosis and the long time-survival of breast cancer. In this context, invasive carcinomas under $10 \mathrm{~mm}$ in size, which most often exhibit a NO-lymph node status, have an excellent 10 -year-tumor-free survival rate of $98 \%$ [1]. With respect to intraductal breast tumors, this rate increases to $100 \%$ if adequate therapy is performed.

Self-examination and medical clinical examination of the breast do not improve lifetime prognosis, because the average size of a palpable tumor is 2 to $3 \mathrm{~cm}$ [2]. Also, sole sonography of the breast is not adequate for the early detection of breast cancer [3]. To date, only the exclusive use of mammography within populationrelated screening programs has been able to demonstrate that a reduction of breast cancer mortality is possible $[4,5]$. The main strength of mammography is the reliable visualization of microcalcifications. These small calcifications can be the first sign of malignancy in non-dense, as well as in dense breast tissue. However, mammography is drastically limited in the detection of tumors that are not associated with microcalcifications in dense breasts. This limitation leads to a decrease of sensitivity of mammography to $30-40 \%$ in women with density type c or d according to ACR [6]. In this constellation even sonography of the breast is superior to mammography for the early detection of carcinomas $[6,7]$. 
It is uncontroversial that quality assured MRI of the breast is superior to all other imaging modalities for the early detection of breast cancer. Several studies demonstrate the superiority and the high value of MRI in women with defined high risk profile [7-9]. There is consensus that about $95 \%$ or more of all carcinomas of the breast are detectable on MRI. The remaining 5\% of lesions that were not seen on MRI were intraductal tumors or very small invasive carcinomas depicted on mammography due to the associated microcalcifications [7-9]. Clinical examination and ultrasound do not give any additional information. Several studies demonstrate, that about $50 \%$ of all carcinomas would not be depicted in a diagnostic concept without MRI [7-9].

The purpose of this study was to evaluate qualityassured high resolution MRI of the breast performed at consecutive intervals of 24 months or less in asymptomatic women and patients with respect to the questions: Does repetitive performed exclusive MRI allow a reliable detection of breast cancer at an early stage regarding the tumor size and stage at the time of diagnosis, and does it possibly make other imaging modalities dispensable.

\section{Material and Methods}

This evaluation included consecutive women and patients, who had a normal MRI of the breast (MR BIRADS 1,2,3) and one or more follow-up MRI exams at an interval of 24 months or less. Women with a suspicious finding (MR BIRADS 4 or 5 ) on the initial MRI examination, or with a follow-up MRI exam at an interval of more than 24 months were excluded from the study. All women in this study were asymptomatic. They referred for screening MRI or had a personal history of breast cancer and were referred for a follow-up MRI. In the screening group, the degree of familial risk was categorized as mild, moderate, or high. Life-time risk was defined as "mild" if there was no breast and/or ovarian cancer in the womans personal medical history or that of her first-degree relatives. Criteria of "high" risk were a positive genetic testing (i.e. BRCA 1, BRCA 2 ), more than three first-degree relatives with breast and/or ovarian cancer, personal history of a biopsy with histological results associated with an increased breast cancer risk (i.e. LIN, $A D H$ ), and patients after irradiation therapy of the chest region. Women, who were not placed in one of these two categories, were classified to have a "moderate" risk profile. Patients with a personal history of breast cancer were subdivided into those who had undergone breast conserving therapy (BCT) or mastectomy (ME).

All MRI-examinations were performed on a 1.5Tesla whole-body scanner Signa HDX (Fa. GE Healthcare, Milwaukee, WI, USA) using a dedicated open 4-channelbreast surface coil (Fa. MRI Device, Knaresborough, NY, USA) with an integrated device for the immobilization of both breasts in cranio-caudal orientation (Fa. Noras, Hoechberg, Germany). Premenopausal women were examined between days 7 and 14 of their menstrual cycle. The MRI examination included a fat-saturated T2 weighted inversion-recovery-sequence with the following parameters: repetition time (TR)/ echo time (TE) $6925 \mathrm{~ms} / 67 \mathrm{~ms}$, field-of-view (FOV) $380 \mathrm{~mm}, 50$ slices of 2.0-2.5 mm, matrix of $512 \times 512$, and a measurement time of 4:51 minutes. Subsequently, 3D T1 weighted gradient-echo-sequences were performed repetitively (once before, and 5 times after administration of contrast material) using the following parameters: TR/TE $8.4 \mathrm{~ms} /$ $4.1 \mathrm{~ms}$, FOV $380 \mathrm{~mm}, 50$ slices of $2.0-2.5 \mathrm{~mm}$, Matrix $512 \times 512$ without interpolation, and a measurement time of $86 \mathrm{~s}$ per sequence. The phase encoding gradient (PEG) was in the fronto-dorsal orientation for the first, and in the medio-lateral orientation for the second precontrast measurement. After administration of contrast material, two measurements followed with the PEG in the medio-lateral orientation, and one measurement with the PEG in the fronto-dorsal orientation. From May 2003 to December 2012 Gd-DTPA (Magnevist ${ }^{\circledR}$, Schering company, Berlin, Germany/Magnevist ${ }^{\circledR}$, Bayer company, Leverkusen, Germany) was administered at a dosage of $0.1 \mathrm{mmol}$ per $\mathrm{kg}$ body weight via an automatic injector (flow $3 \mathrm{ml} / \mathrm{second}$ ) to a peripheral vein. From January 2013 to the end of the study, a cyclic contrast material (Gadovist ${ }^{\circledR}$, Bayer company, Leverkusen, Germany) was administered in an equivalent dosage. In all cases $20 \mathrm{ml}$ of physiologic saline solution was applied after application of the contrast material to wash out the infusion tubing.

Postprocessing included the subtraction of the precontrast images from the first and second postcontrast measurements with the PEG in the mediolateral as well as in the fronto-dorsal orientation on a pixel-to-pixel basis. In addition, maximum-intensityprojections (MIP) of these subtraction images and the T2-images were performed [10].

The evaluation of the MR images included the separate BIRADS assessment for each breast according to the information given in the medical report. Images were assessed in 5 categories: (MR-BIRADS 1: Negative finding; MR-BIRADS 2: Benign finding; MR-BIRADS 3: Probably benign finding; MR-BIRADS 4: Suspicious abnormality; MR-BIRADS 5: Highly suggestive of malignancy) [11]. To calculate the sensitivity and specificity, MR-BIRADS 1-3 were classified as "benign", and MR-BIRADS 4 and 5 were classified as "malignant" lesions.

The breast density type was categorized depending on the amount of fibroglandular tissue (FGT) on mammography or in the T1-weighted precontrast images. Four density types were differentiated according to the recommendations of the ACR (density-type a: Almost entirely fat; density-type b: Scattered fibroglandular tis- 
sue; density-type c: Heterogeneous fibroglandular tissue; density-type d: Extreme fibroglandular tissue) [11].

Mammography and/or breast ultrasound were only regarded in women with lesions categorized as BIRADS 4 or 5 in MRI. These women underwent a bilateral digital full-field-mammography on a Senographe 2000D (GE Healthcare, Milwaukee, WI, USA) in one (mediolateral oblique, MLO) or in two (MLO plus cranio-caudal, CC) views. Mammograms from external facilities were not included. The density type (a-d) and the BIRADScategorizations (MX-BIRADS 1-5) of the mammograms were taken from the primary medical report. A reevaluation of the mammograms was not performed. Ultrasound was done in hand-held technique with a system Logic 9 or Logic 9e using a receiving transducer of 10-15 mHz (GE Healthcare company, Milwaukee, WI, USA). Ultrasound was performed as part of the primary breast imaging and/or as a second-look sonography in full awareness of the MRI findings. The evaluation of the ultrasound examination was performed according to the US-BIRADS- categorization (US-BIRADS 1-5) [11].

\section{Results}

From June 2003 to May 2016 a total number of 2333 women and patients underwent $8975 \mathrm{MRI}$ of the breast with a maximum interval of 24 months between the examinations. MRI was performed for breast cancer screening in 1752 asymptomatic women with different life-time risk profiles (normal risk $n=979$, moderate risk $\mathrm{n}=691$, high risk $\mathrm{n}=82$ ). 581 patients underwent MRI of the breast during the follow up period after breast cancer treatment (breast conserving therapy $n=421$, mastectomy $n=160$ ).

The age of the examined women ranged from 20 to 86 years (average age: 53.0 years) on the date of the first MRI examination. The average density type on mammography was 3.4 according to the classification of the American College of Radiology (type a: 18; type b: 232; type c: 892; type d: 1191).

Without exception, all findings on the first MRI examination of the breast (2333 exams) were classified as BIRADS 1,2 , or 3 , as defined in the inclusion criteria. There were 6642 follow-MRI-exams with intervals of 24 months or less. The number of follow-MRI-exams per person ranged from 1 to 17 examinations corresponding to 2.85 rounds per woman/patient. Follow-up-MRI demonstrated a benign finding correlating to the category BIRADS 1,2, or 3 in 6.352 exams (95.6\%). In the remaining 290 examinations in 272 patients, MRI showed a lesion classified as MR-BIRADS 4 or 5 . All of the 290 lesions were verified by percutaneous biopsy. In this group, 14 patients had two biopsies, and 2 patients had 3 biopsies at different times. Thus, the biopsy rate was $11.7 \%$ per women (4.37\% per exam) within the follow-up MRI examination period. There was a direct correlation between the biopsy rate and the individual life-time risk for breast cancer (Table 1 and Table 2).

After percutaneous biopsy, histology revealed benign lesions (histological category B1 und B2) in 151/290 $(52.1 \%)$ of cases. Lesions with unknown biological potential (B3) were found in 24 (8.3\%) cases. 115/290 $(39.6 \%)$ of the suspicious lesions were malignant (categories B5a, B5b).

Follow-up examinations 6-12 months after biopsy were performed in women with histologically proven benign findings. Thereafter, routine MRI for early detection of breast cancer was performed in intervals of 1-2 years, depending on the individual risk profile. Control MRI within these intervals did not show any progress of a lesion that was biopsied before.

The 24 histological verified B3-lesions were atypical ductal hyperplasia $(A D H, n=2)$, lobular intraepithelial neoplasia (LIN, $n=6$ ), papillary lesions $(n=12)$, phylloid tumors $(n=2)$, and radial scars $(n=2)$. Following the recommendation of the interdisciplinary conference, open biopsy was performed in 12 of these cases. None of these open biopsies, however, showed malignancy.

All patients with a histologically proven malignant tumor on percutaneous biopsy (B5a, B5b) underwent subsequent open biopsy. Histology revealed 36/115 intraductal carcinomas, subclassified as low grade DCIS in 11 , intermediate type DCIS in 16 , and high grade DCIS in 9 cases. The extent of DCIS ranged from $5 \mathrm{~mm}$ to 61 $\mathrm{mm}$ (average size $16.6 \mathrm{~mm}$ ). Four patients with DCIS had additional sentinel-lymphadenectomy due to the large size of the lesion. The nodal state of all of these patients was negative.

The other 79/115 cases with open biopsy had a histologically verified invasive breast cancer. These were invasive ductal carcinomas (IDC, $n=61$ ), invasive lobular carcinomas (ILC, $\mathrm{n}=11$ ), tubular carcinomas (TC, $n=6)$, and one mucinous breast cancer (MC, $n=1)$. The tumor sizes were pT1a in 17 (21.5\%) cases, pT1b in 39 $(49.4 \%)$ cases, and pT1c in $22(27.8 \%)$ cases. Only one invasive carcinoma (1.3\%) was larger than $2 \mathrm{~cm}$ (pT2). The average size of all invasive breast cancers was 9.0 $\mathrm{mm}$ (range: $2 \mathrm{~mm}$ - $22 \mathrm{~mm}$ ). The average size of TC was $5.5 \mathrm{~mm}$, of IDC $8.7 \mathrm{~mm}$, and of ILC $12.5 \mathrm{~mm}$. Dependent on the grading, invasive G1-carcinomas had an average size of $7.3 \mathrm{~mm}, \mathrm{G} 2$-carcinomas of $8.6 \mathrm{~mm}$, and G3carcinomas of $10.9 \mathrm{~mm}$. The correlation between the time intervals between MRI examinations and the tumor size, histopathological subtype, and grading is presented in Figure 1.

In 74/79 cases with invasive breast cancer lymphadenectomy was performed. The remaining 5 patients had no axillary lymph nodes removed due to a previous axillary dissection. Histology revealed $71 / 74$ (95.9\%) cases with normal lymph nodes and three patients with a positive state of the lymph node involvement (pNmic, pN1a, pN2a). 


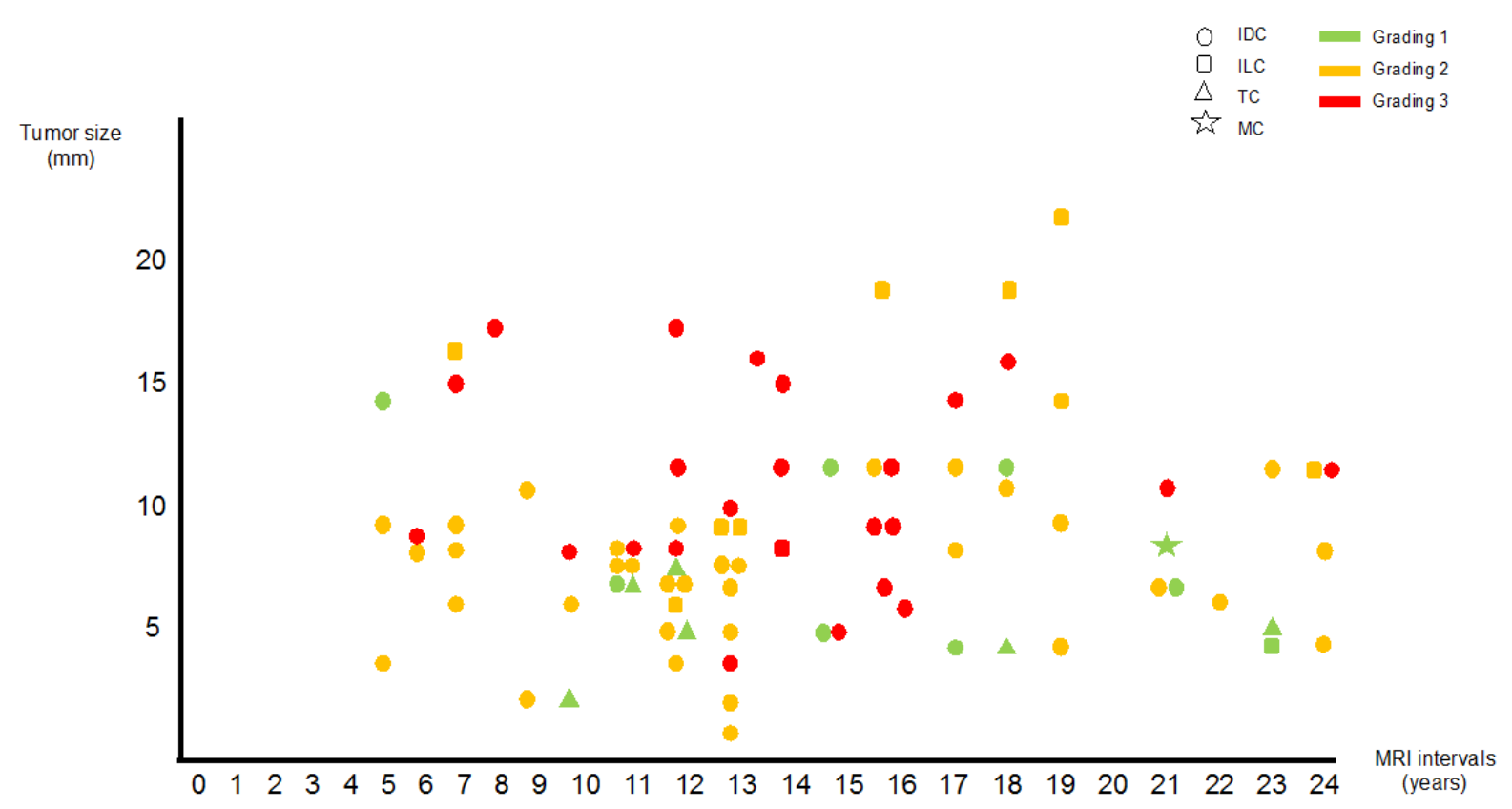

Figure 1: Invasive breast carcinomas. Tumor size (mm), tumor histology (IDC, ILC, TC, MC), and grading $(1,2,3)$ depending on the time interval between the MRI examinations (in months).

Within the study group, 2 patients came ahead of the recommended schedule because of a palpable mass in the breast. Both patients had an invasive breast cancer of $8 \mathrm{~mm}$. In one case, the patient felt a palpable mass in the upper inner quadrant of the breast after reducing her body weight by $12 \mathrm{~kg}$. In the other case, a patient with implants noticed a palpable mass in the areolar region. Regarding the total number of malignant tumors, this corresponds to a rate of interval breast cancer of $1.7 \%$ in this study.

Patients with high risk profile and those with treated breast cancer had a significantly higher detection rate of breast cancer (12.2\% rsp. $7.7 \%$ ) compared to women with normal or moderately increased risk profile (3-4\%). A detailed list of the results is given in Table 1.

In the group of patients with breast carcinoma detected in MRI, six patients refused mammography for personal reasons. In the remaining group of 109 patients, mammography missed $87 / 109$ (79.1\%), and ultrasonography missed 73/115 (63.5\%) of the tumors. The combination of mammography and ultrasound missed $62 / 115$ (53.9\%) of all malignant tumors. Of the 42 carcinomas detected with ultrasound, $21.4 \%$ were depicted retrospectively (second look) (Table 2). The main cause of this limited value of mammography in the detection of breast cancer has to be seen in the high parenchymal density in the women/patients: The average density type in this study was 3.4 according to the initial classification of the American College of Radiology. In this constellation even sonography of the breast is superior to mammography for the early detection of carcinomas $[6,7]$.

\section{Discussion}

To date there are many publications reporting on the value of different imaging modalities for the early detection of breast cancer in asymptomatic women. Numerous studies demonstrates the value of mammography in population-based screening programs $[4,5,12]$. Various other studies compare the value of different imaging modalities (mammography, breast ultrasound and MRI of the breast) in high-risk women $[7-9,13,14]$ as well as in normal-women [15]. All of these studies base their evaluation on the results of the initial and of the follow up examinations. This study differs in its study design, because all women and patients included in this study had an inconspicuous MRI of the breast (BIRADS $1,2,3)$ on the initial examination. Thus, only the results of subsequent routine breast MRI exams performed within an interval of 2 years or less were evaluated. The goal of this study is to ascertain whether or not routine MRI of the breast at intervals of 24 months or less leads to a significant reduction of tumor size and stage at the time of diagnosis.

The results of this study demonstrate that MRI of the breast does allow indeed the detection of breast cancer at an early tumor stage when performed repetitively at intervals of 24 months or less. In $80 \%$ of patients with breast carcinomas, MRI depicted the tumor at an early histological stage (pTis, pT1a, or pT1b) with an excellent prognosis. In high risk groups, Kriege, et al., Riedl, et al., and Kuhl, et al. reported a detection rate for DCIS and invasive breast cancer smaller than $11 \mathrm{~mm}$ in breast MRI of $50 \%, 72 \%$, and $74 \%$ [7-9]. In this context, the rate of detected breast cancer in the presented evaluation are very similar to the results reported by Kuhl, et al. [7]. In comparison to these data, the rate of early breast cancers (pTis, pT1a, or pT1b) depicted in quality-assured mammography screening programs ranges from 50 to $60 \%[12]$. 
Table 1: Cohort depending on the individual risk profile (age, MRI, BIRADS, histology, lymph node stage).

\begin{tabular}{|c|c|c|c|c|c|c|}
\hline & & High risk & $\begin{array}{l}\text { Status with } \\
\text { BCT or ME }\end{array}$ & $\begin{array}{l}\text { Moderate } \\
\text { risk }\end{array}$ & $\begin{array}{l}\text { Normal } \\
\text { risk }\end{array}$ & Total \\
\hline Women & Number & 82 & 581 & 691 & 979 & 2333 \\
\hline Age & Years & 44.4 & 56.6 & 51.1 & 53.0 & 53.0 \\
\hline Range of age (min-max) & Years & $20-80$ & $28-86$ & $22-79$ & $27-84$ & $20-86$ \\
\hline Density type (1-4) & Average & 3.61 & 3.12 & 3.46 & 3.49 & 3.39 \\
\hline MRI of the breast & Number & 388 & 2748 & 2569 & 3270 & 8975 \\
\hline Follow-up MRI & Number & 302 & 2171 & 1878 & 2291 & 6642 \\
\hline Follow-up MRI per women & Number & 3.7 & 3.7 & 2.7 & 2.3 & 2.8 \\
\hline Women with MR-BIRADS 1,2,3 & Number & 56 & 493 & 626 & 886 & $\begin{array}{l}2061 \\
(88.3 \%)\end{array}$ \\
\hline Women with MR-BIRADS 4,5 & Number & 23 & 91 & 65 & 93 & $272(11.7 \%)$ \\
\hline Follow-up: MR-BIRADS 1,2,3 & Number & 277 & 2071 & 1807 & 2197 & $\begin{array}{l}6352 \\
(95.6 \%)\end{array}$ \\
\hline Follow-up: MR-BIRADS 3,4 & Number & 25 & 100 & 71 & 94 & $290(4.4 \%)$ \\
\hline MR-BIRADS 4,5 per women & $\%$ & 28.0 & 15.7 & 9.4 & 9.5 & 11.7 \\
\hline MR-BIRADS 4,5 per follow up & $\%$ & 9.0 & 4.6 & 3.8 & 4.1 & 4.4 \\
\hline Histology: B 1,2 & Number & 13 & 51 & 40 & 47 & $151(52.1 \%)$ \\
\hline Histology: B 3 & Number & 2 & 4 & 7 & 11 & $24(8.3 \%)$ \\
\hline Histology: B 5 & Number & 9 & 45 & 23 & 36 & $115(39.6 \%)$ \\
\hline Subtype: B 5a & Number & 3 & 15 & 6 & 12 & 36 \\
\hline Subtype: B 5b & Number & 7 & 30 & 18 & 24 & 79 \\
\hline $\begin{array}{l}\text { MRI interval (in average) in case of } \\
\text { breast cancer }\end{array}$ & Months & 14.22 & 14.62 & 16.01 & 14.13 & 14.95 \\
\hline Carcinoma & Number & 10 & 45 & 24 & 36 & $115(100 \%)$ \\
\hline Carcinoma per women & $\%$ & 12.2 & 7.7 & 3.5 & 3.7 & 4.9 \\
\hline Intraductal carcinomas & Number & 3 & 15 & 6 & 12 & $36(31.3 \%)$ \\
\hline DCIS G1 & Number & 1 & 4 & 2 & 4 & 11 \\
\hline DCIS G2 & Number & 1 & 9 & 3 & 3 & 16 \\
\hline DCIS G3 & Number & 1 & 2 & 1 & 5 & 9 \\
\hline Invasive carcinomas & Number & 7 & 30 & 18 & 24 & $79(68.7 \%)$ \\
\hline IDC & Number & 7 & 22 & 11 & 21 & 61 \\
\hline ILC & Number & 0 & 5 & 4 & 2 & 11 \\
\hline MC & Number & 0 & 1 & 0 & 0 & 1 \\
\hline TC & Number & 0 & 2 & 3 & 1 & 6 \\
\hline pT1a & Number & 2 & 9 & 2 & 4 & 17 \\
\hline pT1b & Number & 4 & 16 & 8 & 11 & 39 \\
\hline pT1c & Number & 1 & 5 & 7 & 9 & 22 \\
\hline pT2 & Number & 0 & 0 & 1 & 0 & 1 \\
\hline Luminal A & Number & 2 & 10 & 9 & 6 & 27 \\
\hline
\end{tabular}




\begin{tabular}{|c|c|c|c|c|c|c|}
\hline Luminal B & Number & 2 & 15 & 6 & 16 & 40 \\
\hline HER & Number & 1 & 0 & 0 & 0 & 1 \\
\hline Triple Negative & Number & 2 & 5 & 2 & 2 & 11 \\
\hline Lymph node negative & Number & 7 & 25 & 18 & 21 & $\begin{array}{l}71 / 74 \\
(95.9 \%)\end{array}$ \\
\hline Lymph node positive & Number & 0 & 0 & 0 & 3 & $3 / 74(4.1 \%)$ \\
\hline No lymphadenectomy & Number & 0 & 5 & 0 & 0 & 5 \\
\hline
\end{tabular}

Table 2: Results of MX and US depending on risk profile and tumor stage.

\begin{tabular}{|c|c|c|c|c|c|}
\hline & High risk & Status with BCT or ME & Moderate risk & Normal risk & Total \\
\hline Malignant tumors, all & 10 & 45 & 24 & 36 & 115 \\
\hline MX n A & 2 & 0 & 1 & 3 & $6(5.2 \%)$ \\
\hline MX R+ & 1 & 8 & 3 & 10 & $22 / 109(20.2 \%)^{*}$ \\
\hline MX F- & 7 & 37 & 20 & 23 & $87 / 109(79.8 \%)^{*}$ \\
\hline US R+ & 5 & 10 & 12 & 15 & $42 / 115(36.5 \%)$ \\
\hline US F- & 5 & 35 & 12 & 21 & $73 / 115(63.5 \%)$ \\
\hline MX+ US R+ & 6 & 16 & 12 & 19 & $53(45.2 \%)$ \\
\hline MX+ US F- & 4 & 29 & 12 & 17 & $62(53.9 \%)$ \\
\hline DCIS & 3 & 15 & 6 & 12 & 36 \\
\hline MX R+ & 0 & 5 & 0 & 4 & $9(25.0 \%)^{* *}$ \\
\hline MX F- & 2 & 10 & 6 & 7 & $25(69.4 \%)^{\star \star}$ \\
\hline US R+ & 1 & 0 & 0 & 4 & $5(13.9 \%)$ \\
\hline US F- & 2 & 15 & 6 & 8 & $31(86.1 \%)$ \\
\hline MX+ US R+ & 1 & 5 & 0 & 7 & $13(\%)$ \\
\hline MX+ US F- & 2 & 10 & 6 & 5 & $23(\%)$ \\
\hline pT1a & 2 & 9 & 2 & 4 & 17 \\
\hline MX R+ & 0 & 0 & 0 & 1 & $1(5.9 \%)^{* *}$ \\
\hline MX F- & 1 & 9 & 2 & 2 & $14(82.4 \%)^{* *}$ \\
\hline US R+ & 1 & 3 & 1 & 2 & $7(41.2 \%)$ \\
\hline US F- & 1 & 6 & 1 & 2 & $10(58.8 \%)$ \\
\hline MX+ US R+ & 1 & 3 & 1 & 2 & $7(41.2 \%)^{* *}$ \\
\hline MX+ US F- & 0 & 6 & 1 & 2 & $9(52.9 \%)^{* *}$ \\
\hline pT1b & 4 & 16 & 8 & 11 & 39 \\
\hline MX R+ & 0 & 1 & 2 & 1 & $4(10.5 \%)^{* \star *}$ \\
\hline MX F- & 4 & 15 & 5 & 10 & $34(89.5 \%)^{* * *}$ \\
\hline US R+ & 2 & 6 & 5 & 2 & $15(38.5 \%)$ \\
\hline US F- & 2 & 10 & 3 & 9 & $24(61.5 \%)$ \\
\hline MX+ US R+ & 2 & 6 & 5 & 2 & 16 (41.0\%) \\
\hline MX+ US F- & 2 & 10 & 3 & 9 & $23(59.0 \%)$ \\
\hline pT1c & 1 & 5 & 7 & 9 & 22 \\
\hline MX R+ & 1 & 2 & 1 & 4 & $8(38.1 \%)^{\star \star \star}$ \\
\hline MX F- & 0 & 3 & 6 & 4 & $13(61.9 \%)^{\star * *}$ \\
\hline US R+ & 1 & 1 & 5 & 7 & $14(63.6 \%)$ \\
\hline US F- & 0 & 4 & 2 & 2 & $8(36.4 \%)$ \\
\hline MX+ US R+ & 1 & 2 & 5 & 8 & $16(72.7 \%)$ \\
\hline
\end{tabular}




\begin{tabular}{|l|l|l|l|l|l|}
\hline MX+ US F- & 0 & 3 & 2 & 1 & $\mathbf{6}(\mathbf{2 7 . 3 \% )}$ \\
\hline pT2 & & & & & \\
\hline MX R+ & 0 & 0 & 1 & 0 & $\mathbf{1}$ \\
\hline MX F- & 0 & 0 & 0 & 0 & $\mathbf{0}(\mathbf{0 . 0} \%)$ \\
\hline US R+ & 0 & 0 & 1 & 0 & $\mathbf{1}(\mathbf{1 0 0 . 0 \% )}$ \\
\hline US F- & 0 & 0 & 1 & 0 & $\mathbf{1}(\mathbf{1 0 0 . 0} \%)$ \\
\hline MX+ US R+ & 0 & 0 & 0 & 0 & $\mathbf{0}(\mathbf{0 . 0} \%)$ \\
\hline MX+ US F- & 0 & 0 & 1 & 0 & $\mathbf{1}(\mathbf{1 0 0 . 0} \%)$ \\
\hline
\end{tabular}

"MX not performed in 6 cases; " $\mathrm{MX}$ not performed in 2 cases; "** $\mathrm{MX}$ not performed in 1 case.

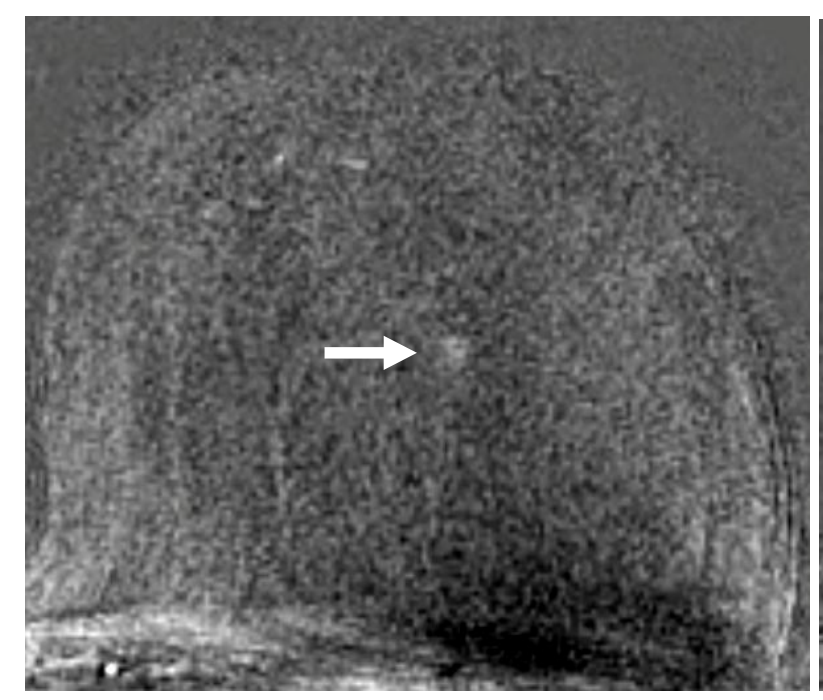

$2 \mathrm{a}$

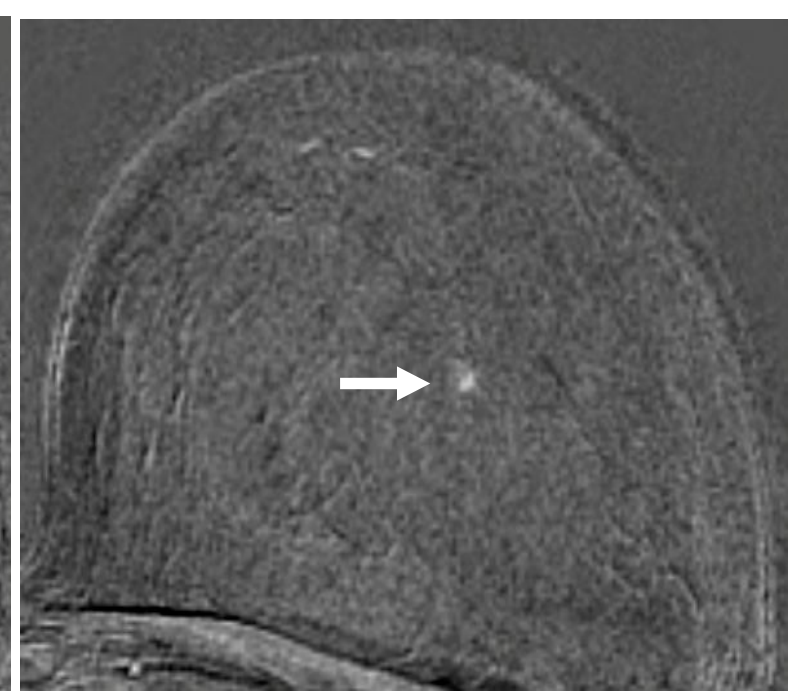

$2 \mathrm{~b}$

Figure 2: a) 65-year-old patient after breast-conserving therapy of the right breast. In the single-slice subtraction image mild focal enhancement in the central part of the left breast. Categorization: MR-BIRADS left breast 2 . Next MRI in 12 months recommended; b) MRI of the breast 13 months later. In the single-slice subtraction image now 3 mm single focus in the central part of the left breast. Categorization: MR-BIRADS left breast 4. No corresponding findings in mammography or ultrasound. MR-guided vacuum biopsy: B5b. Final histology: Invasive ductal carcinoma, pT1a (3 mm), grading 2, Luminal B, pN0.

MRI depicted invasive breast carcinomas at an average size of $9 \mathrm{~mm}$ when performed in intervals of 24 months or less. This size correlates very well with the commonly accepted data concerning the rate of growth of invasive breast cancer, assuming a tumorduplication-time of 200-400 days depending on the aggressiveness of the lesion. An invasive breast cancer of $2 \mathrm{~mm}$, for example, is sustained by diffusion, and will not usually be detected on MRI due to the lack of angiogenesis. One year later the estimated size of this lesion is approximately $4 \mathrm{~mm}$, and tumor angiogenesis will have occured. At this time, MRI is often able to depict this tumor due to its increased enhancement. Another year later, at a tumor size of approximately 8 $\mathrm{mm}, \mathrm{MRI}$ should normally detect this tumor reliably. The dynamics of tumor growth is demonstrated in the following exemplary cases from the study (Figure 2a, Figure 2b, Figure 3a, Figure 3b, Figure 4a and Figure 4b).

The size of the detected invasive breast carcinomas ranged from 2 to $22 \mathrm{~mm}$ in this study. The greater proportion detected by MRI at a size of more than 15 $\mathrm{mm}$ within 2 years after an inconspicious MRI exam were lobular ( 4 of 7 breast carcinomas; $57 \%$ ). The reason for this is the indian-file growth pattern of lobular breast cancers, resulting in large tumor sizes with a low count of tumor cells in comparison to the solid growth type of ductal carcinomas (Figure 5 a and Figure $5 b$ ). In this context it could be considered whether the metric of a tumor in millimeters is an optimal criterion for the assessment of invasive lobular carcinomas. There are, however, also invasive ductal carcinoma that increase in size to $15 \mathrm{~mm}$ or more within an intervall of 1-2 years. In the presented study, all of the detected invasive ductal carcinomas of $15 \mathrm{~mm}$ or larger were tumors of grading 3 (Figure $6 \mathrm{a}$ and Figure $6 \mathrm{~b}$ ). In summary, the detection of breast cancer at a stage of pT2 or higher is rare if MRI is performed at intervals of 24 months or less. In the presented study, this rate was $1.3 \%$ of all invasive carcinomas.

This study demonstrates that the excellent results ascribed to MRI of the breast in women with a defined high risk profile can also be achieved for women with a normal or a moderately increased life-time risk pro- 


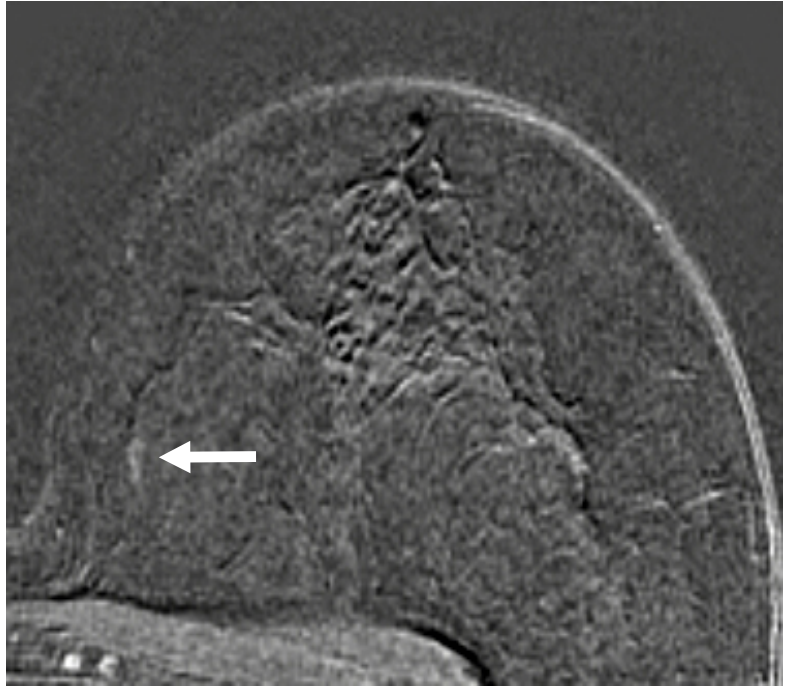

$3 \mathrm{a}$

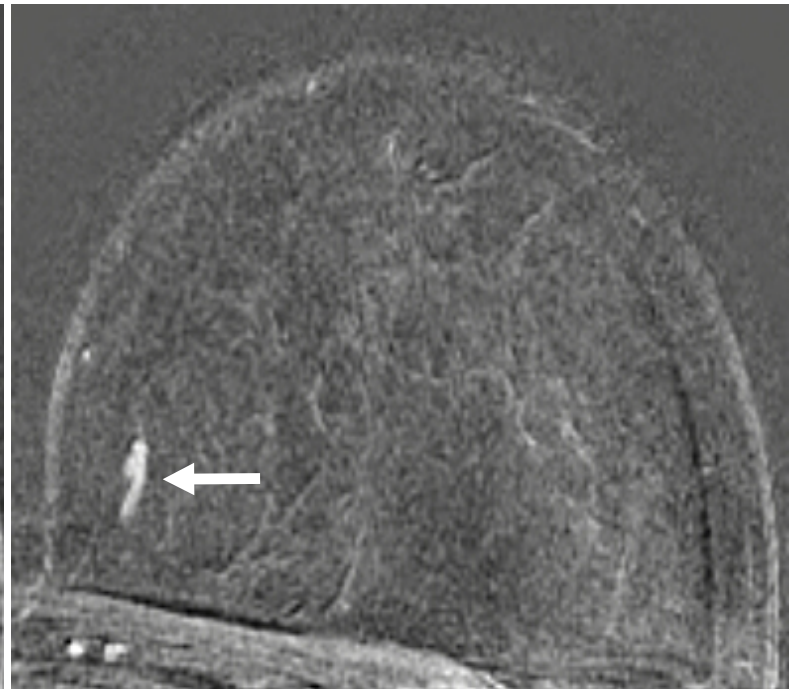

$3 \mathrm{~b}$

Figure 3: a) 65-year-old patient after breast-conserving therapy of the right breast. In the single-slice subtraction image no suspicious enhancement in the inner part of the left breast. Categorization: MR-BIRADS left breast 1 . Next MRI in 12 months recommended; b) MRI of the breast 12 months later. In the single-slice subtraction image now a linear enhancing lesion in the inner quadrant of the left breast. Categorization: MR-BIRADS left breast 4 . No corresponding findings in mammography (MX-BIRADS 1) or ultrasound (US-BIRADS 1). MR-guided vacuum biopsy: B5a. Final histology: DCIS intermediate type G2, pTis $(12 \mathrm{~mm})$, grading 2 .

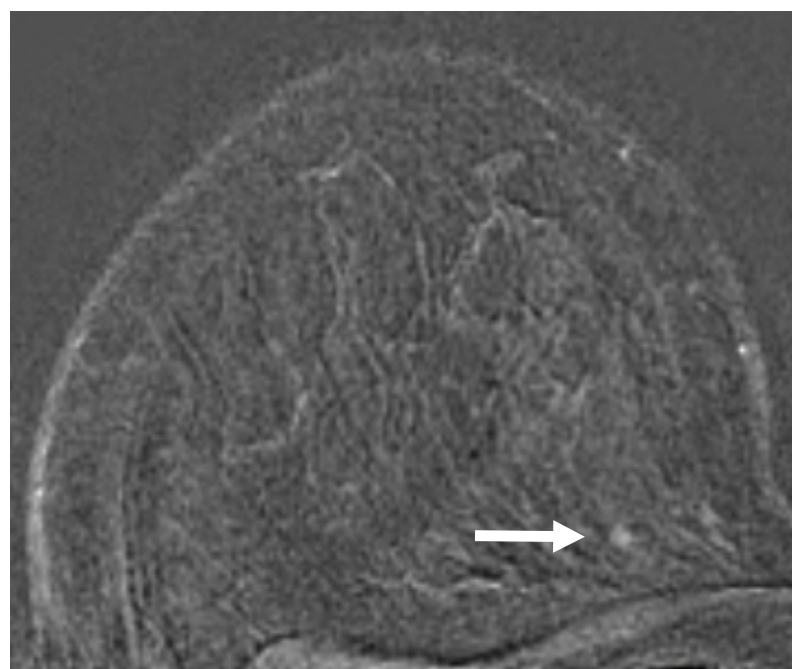

$4 \mathrm{a}$

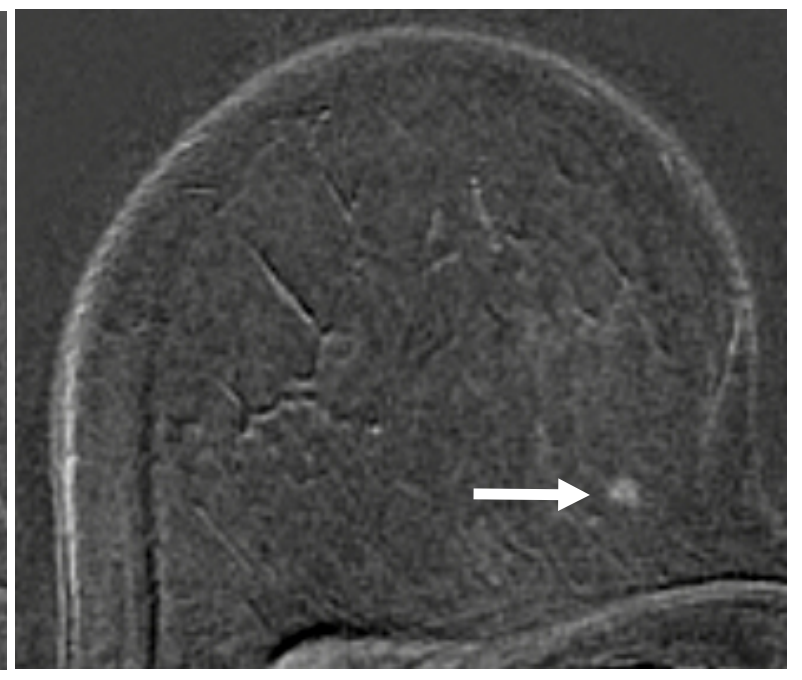

$4 \mathrm{~b}$

Figure 4: a) 62-year-old patient with normal life time risk. In the single-slice subtraction single focus (2 mm) in the inner part of the right breast. Categorization: MR-BIRADS right breast 2. Next MRI was recommended in 18 months; $b$ ) MRI of the breast 24 months later. In the single-slice subtraction image now mild increase of the focus to $4 \mathrm{~mm}$ in the inner quadrant of the left breast. Categorization: MR-BIRADS left breast 4 . No corresponding findings in mammography (MX-BIRADS 1), but semisuspicious lesion in second-look-ultrasound (US-BIRADS 3). US-guided core biopsy: B5b. Final histology: Invasive ductal carcinoma, pT1a (4 mm), grading 2, Luminal A, pN0.

file. Certainly the incidence of breast cancer is higher in women with high risk profile compared to other women with a normal oder moderately increased risk. MRI is superior to all other imaging modalities even in a collective of women with a normal or moderate increased risk profile. This is particulary true for small carcinomas with an excellent prognosis. The detection rate for carcinomas in the stages pTis, pT1a, and pT1b is $16 \%$ for mammography, and $29 \%$ for ultrasound. This rate increases to $39 \%$ when both methods (mammography plus ultra- sound) are combined. In contrast, MRI shows a corresponding detection rate of $98 \%$. It has to be noted, however, that the study collective included predominantly women with a high density type on mammography (average 3.39 according to the old definition of ACR). This means that most of the included women had a density type " $c$ " or " $d$ " according to the new definition. This is a relevant factor influencing the results of mammography. Mammography studies have shown that the sensitivity of mammography decreases to $32-45 \%$ in clients 


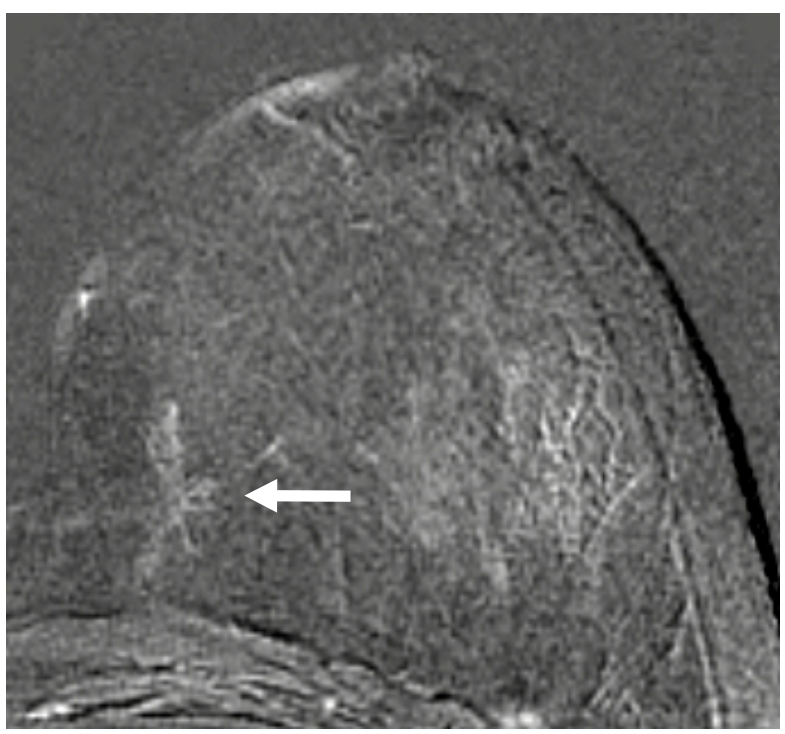

$5 \mathrm{a}$

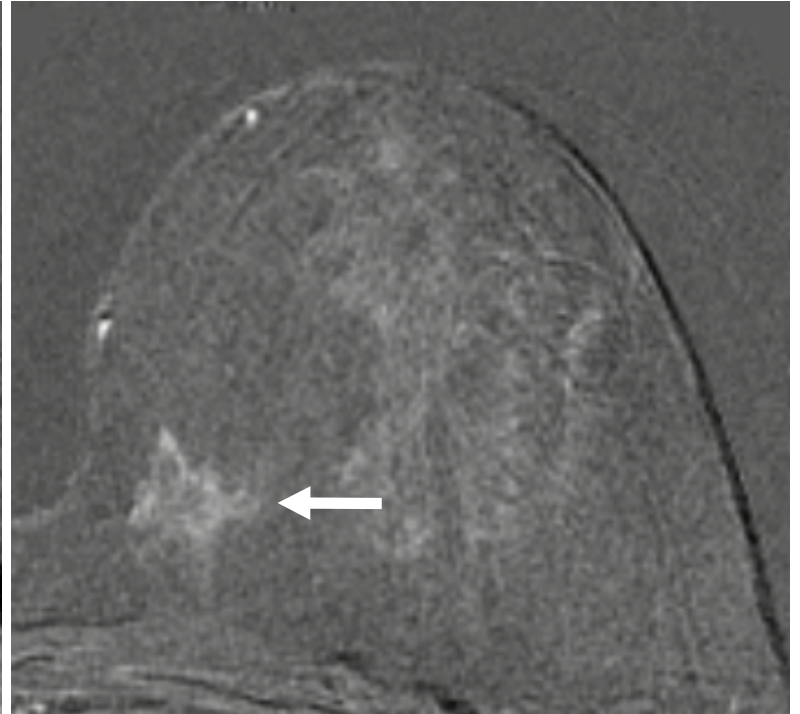

$5 b$

Figure 5: a) 56-year-old patient with moderate increased life time risk. In the single-slice subtraction stellate non-masslike lesion in the inner part of the left breast. Changes were (mis)interpreted as parenchymal enhancement. Categorization: MR-BIRADS left breast 1. Next MRI was recommended in 18 months; b) MRI of the breast 19 months later. Single-slice subtraction image shows an increase of the size of the non-mass-like lesion in the inner quadrant of the left breast to more than $20 \mathrm{~mm}$. Categorization: MR-BIRADS left breast 4. No corresponding findings in mammography (MX-BIRADS 1), but suspicious architectorial distorsion in ultrasound (US-BIRADS 4). US-guided core biopsy: B5b. Final histology: Invasive lobular carcinoma, pT1c (22 mm), grading 2, Luminal A, pN0.

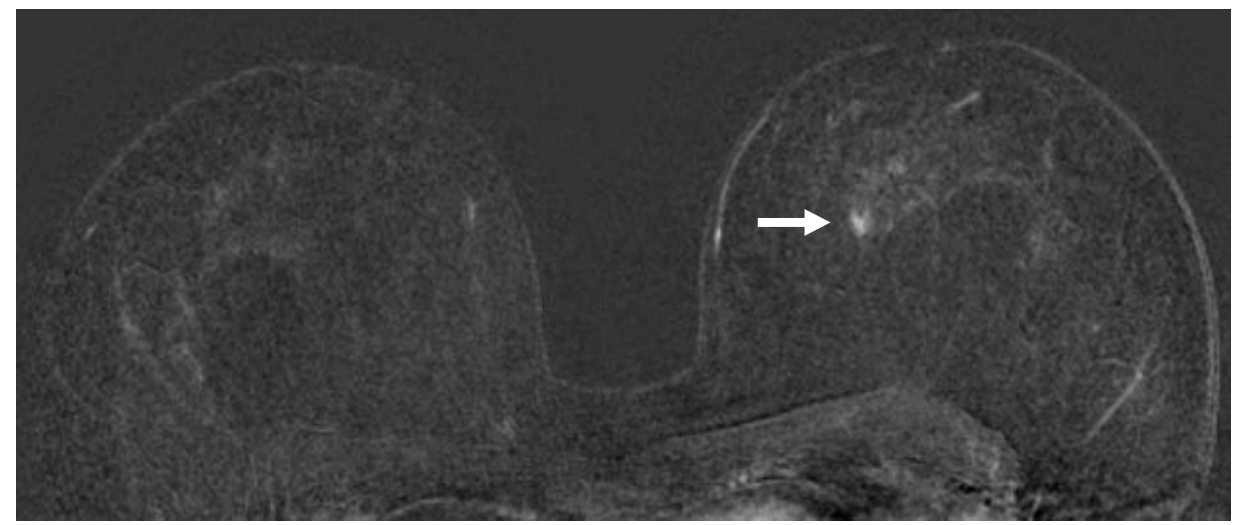

$6 \mathrm{a}$

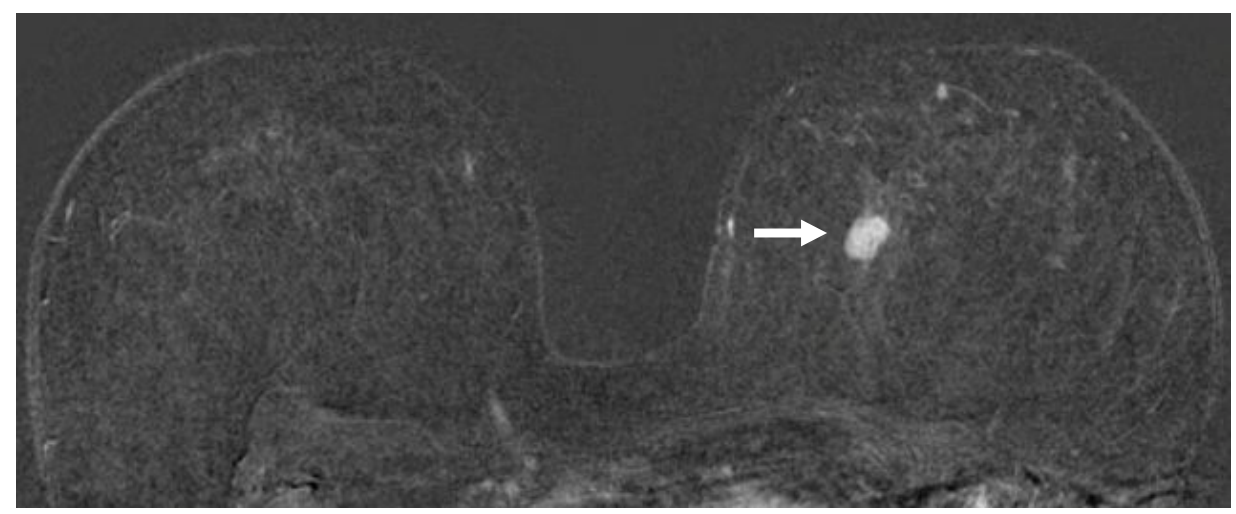

$6 \mathrm{~b}$

Figure 6: a) 70-year-old asymptomatic woman. Normal risk. In the single-slice subtraction image $4 \mathrm{~mm}$ single focus in the central part of the left breast. Normal findings of the right breast. Categorization: MR-BIRADS right breast 1/MR-BIRADS left breast 3. Control-MRI in 6 months recommended; b) Control-MRI of the breasts 7 months later. In the single-slice subtraction image $15 \mathrm{~mm}$ enhancing mass in the central part of the left breast. Normal findings of the right breast. Categorization: MRBIRADS right breast 1/MR-BIRADS left breast 5. Corresponding findings in mammography (MX-BIRADS 4) and ultrasound (US-BIRADS 5). US-guided core biopsy: B5b. Final histology: Invasive ductal carcinoma, pT1c (15 mm), grading 3, Luminal $\mathrm{B}, \mathrm{pN} 0$. 
with high parenchymal density type " $c$ " or "d" $[6,16]$.

The percentage of patients with invasive breast cancer and a negative lymph-node status (pNO) was greater than $95 \%$. This rate is higher than that reported for mammography performed in individual concepts of early detection of breast cancer $(57 \%)$, and that reported for quality assured screening programs $(80 \%)$ [12]. In multimodal screening concepts for women with a high risk profile, this rate ranges from $64 \%$ (HIBCRIT study) [8] to $89 \%$ (EVA study) [7]. The presented data allows the conclusion that MRI performed at intervals of 24 months or less results not only in a reduction of tumor stage, but also significantly decreases the number of detected nodal-positive invasive breast carcinomas. In conclusion, it can be expected that continuous MRI of the breast withi interval of 2 years or less should reduce the mortality of breast cancer more than international screening programs already do. In this context. Continuous MRI of the breast in intervals of 24 months or less would conceivable also reduces medical costs due to the elimination of advanced tumor stages. It is up to economists to calculate the costs of MRI of the breast versus the reduction of treatment costs due to the detection of breast cancer at an earlier stage.

A remainimg problem of continuous MRI of the breast in defined intervals are the costs that are much more higher than the costs of other imaging modalities. First concepts to reduce breast MRI examination costs show promise. Fischer, et al. presented a "first-passconcept" with a total breast MRI measurement time of 4-5 minutes in 2012. Moreover, they inaugurated the idea of a MRI system with 2-3 examination tables to increase the rate of examined women to 6-8 per hour. In combination with a reasonable reduction contrast material costs, they calculated a rate for breast MRI of 240 EURO per examination [17]. This is equivalent to 10 EURO per MRI exam per month at a 24 months interval. The idea of an abbreviated breast MRI protocol has since been evaluated in a clinical study by Kuhl, et al. [7] They showed that a short MRI protocol ( $3 \mathrm{~min}$ ) and an evaluation time of $30 \mathrm{sec}$ per examination result in equally good detection rates as the evaluation of a fulltime MRI examination of 15-20 minutes [18].

As reported earlier, the cost aspect of a populationbased MRI screening for women with dense breast tissue is solvable. A more relevant limitation is the lack of a standardized and quality-assured technique and method, as well as the need to increase the number of experienced reader in order to implement a nationwide use of breast-MRI. These requirements quality assurance and reader's experience - have to be optimized before an area-wide use of MRI of the breast is feasable.

In conclusion, the presented results, as well as the results of abbreviated breast MRI protocols, suggest that MRI of the breast is a suitable and effective screening concept especially for women with high density breast tissue (density types ACR c and d). In this constellation, MRI allows an increase of sensitivity from about $50 \%$ for mammography to approximately $95 \%$ for MRI. This concerns about 25 to $45 \%$ of all women at a breast-cancer relevant age, who have dense breast tissue according to the data of international screening evaluations $[7,18]$.

\section{References}

1. (2013) Stat Bite Age-Adjusted SEER. Breast cancer incidence rates all ages, all races, female (2000-2009). J Natl Cancer Inst 105: 512.

2. Anthony B Miller, Teresa To, Cornelia J Baines, Claus Wall (2002) The canadian national breast screening study-1: Breast cancer mortality after 11 to 16 years of follow-up: A randomized screening trial of mammography in women age 40 to 49 years. Ann Intern Med 137: 305-312.

3. Nothacker M, Lelgemann M, Giersiepen K, Weinbrenner S (2007) Evidenzbericht zur S-3- Leitlinie Brustkrebsfrüherkennung in Deutschland, ed Version 1.00. Berlin, Ärztliches Zentrum für Qualität in der Medizin (ÄZQ), 31.

4. Roberts MM, Alexander FE, Anderson TJ, Chetty U, Donnan PT, et al. (1990) Edinburgh trial of screening for breast cancer: Mortality at seven years. Lancet 335: 241246.

5. Tabar L, Fagerberg G, Chen HH, Duffy SW, Smart CR, et al. (1995) Efficacy of breast cancer screening by age. New results from the Swedish Two-County Trial. Cancer 75: 2507-2517.

6. Pisano ED, Hendrick RE, Yaffe MJ, Baum JK, Acharyya S, et al. (2008) Diagnostic accuracy of digital versus film mammography: Exploratory analysis of selected population subgroups in DMIST. Radiology 246: 376-383.

7. Kuhl CK, Weigel S, Schrading S, Arand B, Bieling $H$, et al. (2010) Prospective multicenter cohort study to refine management recommendations for women at elevated familial risk of breast cancer: The EVA Trial. J Clin Oncol 28: 1450-1457.

8. Sardanelli F, Podo F, Santoro F, Manoukian S, Bergonzi S, et al. (2011) Multicenter surveillance of women at high genetic breast cancer risk using mammography, ultrasonography, and contrast-enhanced magnetic resonance imaging (the high breast cancer risk italian 1 study). final results. Invest Radiol 46: 94-105.

9. Riedl CC, Luft N, Bernhart C, Weber M, Bernathova M, et al. (2015) Triple-modality screening trial for familial breast cancer underlines the importance of magnetic resonance imaging and questions the role of mammography and ultrasound regardless of patient mutation status, age, and breast density. J Clin Oncol 10: 1128-1135.

10. Fischer U (2012) Practical MR Mammography. High Resolution MRI of the Breast. ( $2^{\text {nd }}$ edn), New York, NY: TPS.

11. (2013) American College of Radiology. ACR BI- RADSmagnetic resonance imaging. In: ACR breast imaging reporting and data system atlas. Reston, Va: American College of Radiology, 2013.

12. (2016) Deutsches Mammographie-Screening-Programm: Jahresbericht Evaluation 2013. Kooperationsgemeinschaft Mammographie, Berlin. 
13. Kriege M, Brekelmans CT, Boetes C, Besnard PE, Zonderland $\mathrm{HM}$, et al. (2004) Efficacy of MRI and mammography for breast-cancer screening in women with a familial or genetic predisposition. N Engl J Med 351: 427-437.

14. Passaperuma K, Warner E, Causer PA, Hill KA, Messner S, et al. (2012) Long-term results of screening with magnetic resonance imaging in women with BRCA mutations. $\mathrm{Br} \mathrm{J}$ Cancer 107: 24-30.

15. Fischer U, Luftner-Nagel S, Baum F, Marten-Engelke K, Wienbeck S (2018) The value of quality-assured magnetic resonance imaging of the breast for the early detection of breast cancer in asymptomatic women. J Comput Assist Tomogr 42: 1-5.
16. Berg WA, Gutierrez L, NessAiver MS, Carter WB, Bhargavan $M$, et al. (2004) Diagnostic accuracy of mammography, clinical examination, US, and MR imaging in preoperative assessment of breast cancer. Radiology 233: 830-849.

17. Fischer U, Korthauer A, Baum F, Luftner-Nagel S, Heyden D, et al. (2012) Short first-pass MRI of the breast. Acta Radiol 53: 267-269.

18. Kuhl CK, Schrading S, Strobel K, Schild HH, Hilgers RD, et al. (2014) Abbreviated breast Magnetic Resonance Imaging (MRI): First postcontrast subtracted images and maximum-intensity projection-a novel approach to breast cancer screening with MRI. J Clin Onc 32: 2304-2310. 